\title{
Gratings in novel fibre geometry for applications in shape sensing
}

\author{
H. Dobb ${ }^{\mathrm{a}}$, T. Allsop ${ }^{\mathrm{a}}$, D.J. Webb ${ }^{\mathrm{a}}$, K. Kalli ${ }^{\mathrm{b}}$ \\ ${ }^{a}$ Photonics Research Group, Aston University, Aston Triangle, Birmingham, B4 7ET, UK. \\ ${ }^{\mathrm{b}}$ Higher Technical Institute, C. Kavafi Str., Aglantzia, P.O. Box 20423, 2152 Nicosia, Cyprus.
}

\begin{abstract}
A long period grating (LPG) fabricated in progressive three-layered (PTL) fibre is described. The grating with a period of $391 \mu \mathrm{m}$, had dual attenuation bands associated with a particular cladding mode. The dual attenuation bands have been experimentally characterised for their spectral sensitivity to bending, which resulted in the highest sensitivity to bending seen for this particular fibre and temperature. The spectral characteristics of the fibre have been modelled giving good agreement to the experimental data as well as showing that the attenuation bands are both associated with the second order $\mathrm{HE} / \mathrm{EH}_{2, \mathrm{n}}$ cladding mode.
\end{abstract}

\section{INTRODUCTION}

A Long-Period grating (LPG) is an axially periodic refractive index variation inscribed in the core of a single-mode optical fibre. This index perturbation couples light at discrete wavelengths between the core and cladding modes resulting in attenuation bands in the transmission spectrum of the fibre. This characteristic of LPGs has resulted in many applications for telecommunications including erbium-doped fibre amplifier gain equalisers [1], and band rejection tunable filters [2]. LPGs are also of interest for sensing applications due to spectral shifts of the attenuation bands occurring as a result of their sensitivity to several external measurands: temperature, strain, curvature and surrounding refractive index (SRI) [3-5]. These shifts are strongly dependant on the structure and composition of the fibre as well as the operating wavelength and periodicity of the grating itself. For telecommunications and some sensing applications, one of the major drawbacks of using LPGs is their sensitivity to SRI changes. LPGs fabricated in singlemode progressive three-layered (PLT) fibre have been proven to be insensitive to SRI changes [6]. This property allows the LPGs to be used in applications where the sensor is embedded in a composite material without affecting the transmission spectrum of the grating.

In this paper, we report on investigations of the bending and temperature spectral sensitivities of dual attenuation bands; of a LPG inscribed into PTL fibre. The attenuation bands are associated with a single cladding mode and are located either side of the modal turning point (TP). Attenuation bands located in this area in singlemode two-layered fibre have been proven to exhibit enhanced sensitivity to external measurands [7].

\section{EXPERIMENT AND RESULTS}

The PTL optical fibre was supplied by Fibercore Ltd and was originally designed for a cladding pumped erbium doped fibre laser. Its radii are $r_{\text {core }}=2.6 \mu \mathrm{m}, r_{\text {innercladding }}=45.2 \mu \mathrm{m}$ and $r_{\text {outercladding }}=62.5 \mu \mathrm{m}$. Since the fibre was not designed to be photosensitive it was hydrogenated at a pressure of 170 bar at a temperature of $80{ }^{\circ} \mathrm{C}$ for 70 hours. The LPG was written using a CW frequency doubled argon ion laser at $244 \mathrm{~nm}$, with a point-by-point writing technique. The fabricated LPG had a grating period of $391 \mu \mathrm{m}$ and a length of $7 \mathrm{~cm}$. Characterisation of the dual attenuation bands was carried out by illuminating the grating with a broadband light source and observing the resulting transmission spectrum with an optical spectrum analyser (OSA) with an accuracy of $0.06 \mathrm{~nm}$. Fig.1 shows the spectral profile of the dual attenuation bands, located at $1540 \mathrm{~nm}$ and $1646 \mathrm{~nm}$. The black line shows the transmission spectrum of the LPG in PTL optical fibre spliced to standard SMF. Interference fringes are observed caused by cavities being created between the mismatched layers of fibre, resulting in inaccurate characterisation of the attenuation bands. This effect has been reported in [8]. To significantly reduce this effect, the excess PTL fibre was removed and only the section of fibre containing the LPG spliced into standard single mode fibre (SMF); the transmission spectrum being shown by the grey trace in Fig. 1. 


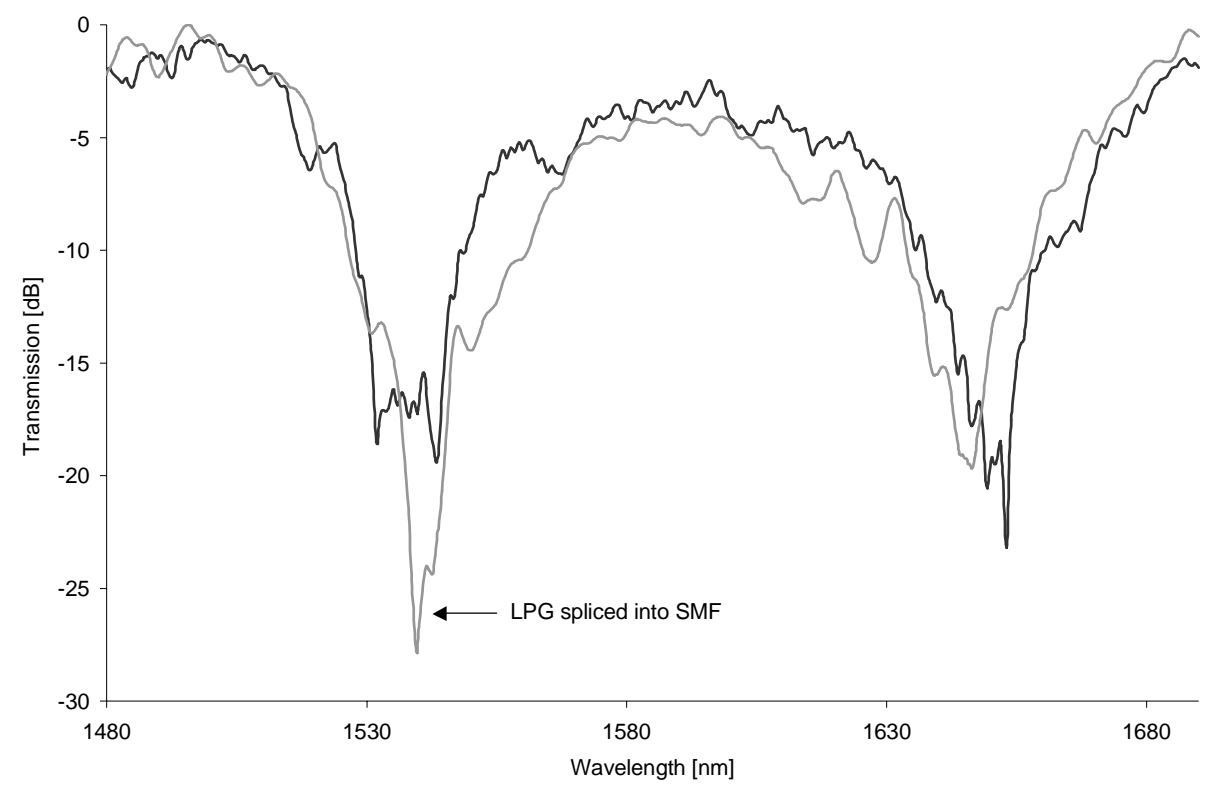

Fig. 1. Spectral profile of the dual attenuation bands with and without the excess PTL fibre removed.

\subsection{SPECTRAL BENDING CHARCTERISTICS}

The bend sensitivity measurements were made by clamping the LPG midway between two blocks, one of which was mounted on a translation stage capable of bending the fibre; see Fig. 2.

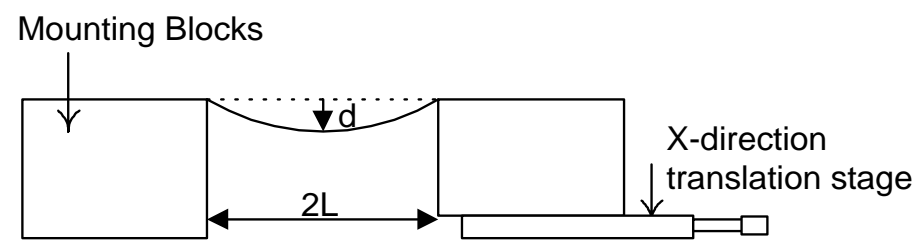

Fig. 2. Schematic of the bending rig.

When the LPG is midway between the two blocks, the resulting curvature, $\mathrm{R}$, of the sensor is given by [9]

$$
R=\frac{2 d}{d^{2}+L^{2}}
$$

where $\mathrm{d}$ is the bending displacement and $\mathrm{L}$ is half the distance between the fibre clamping points. With increasing curvature the band centred at $1540 \mathrm{~nm}$ experienced a red wavelength shift and the band centred at $1646 \mathrm{~nm}$ experienced a blue wavelength shift. The bands continue to move together until they converge. At this point they are located exactly at the TP of the modal phase matching curve. With a continued increase in curvature the band disappears, which occurs at a curvature of $2.61 \mathrm{~m}^{-1}$, see Fig. 3 . 


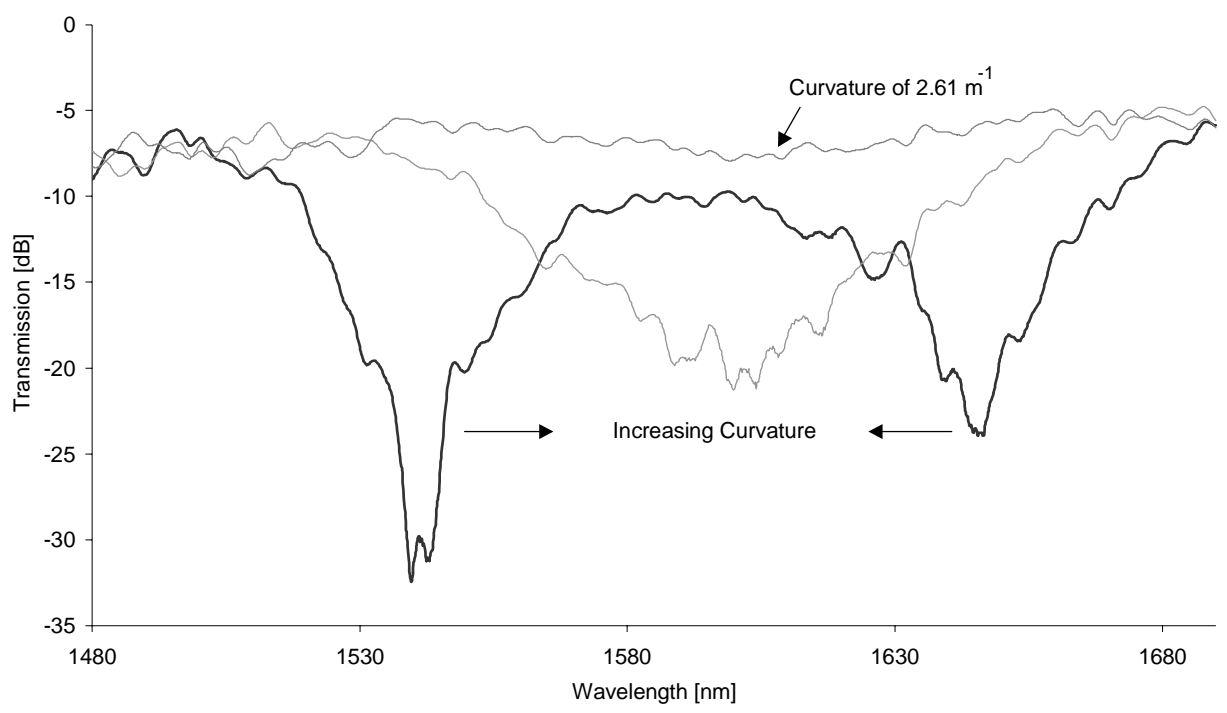

Fig. 3. Convergence of the attenuation bands with increasing curvature.

The resulting bend sensitivity can be seen in Fig. 4.

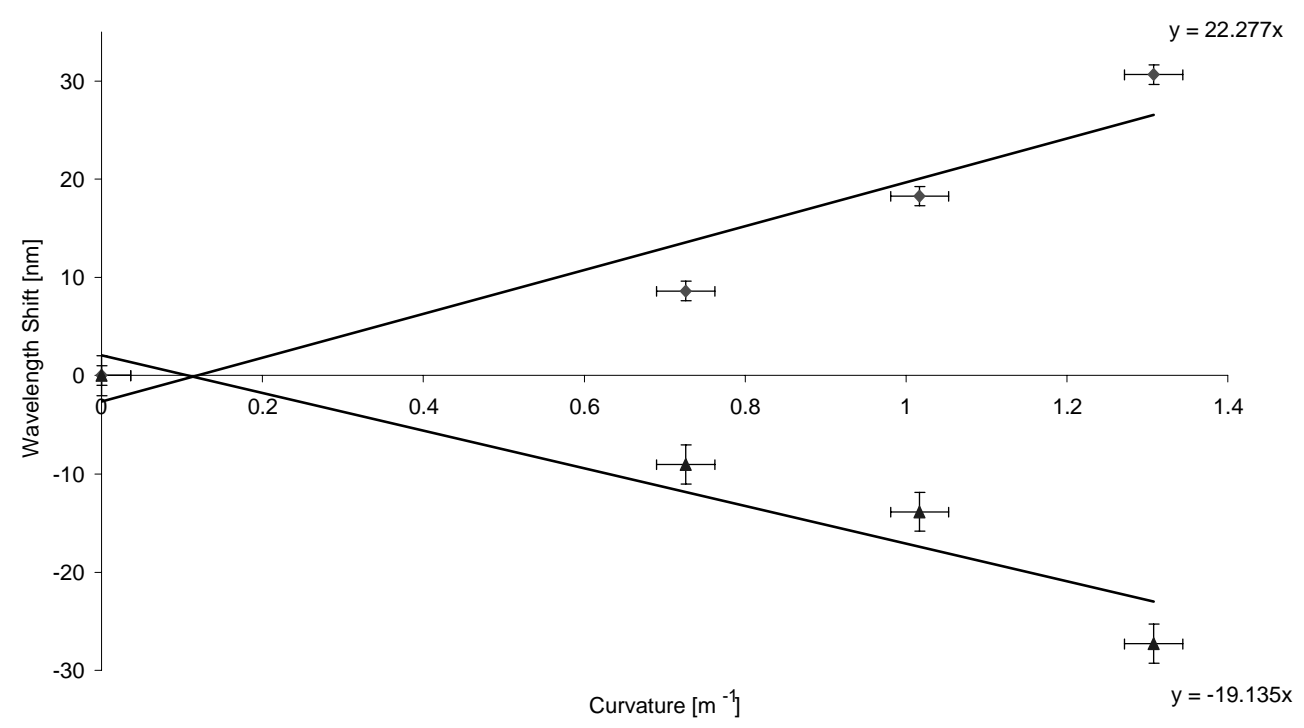

Fig. 4. Bend sensitivity of the attenuation bands of the LPG at 1540/1640nm.

The bend sensitivity is determined by the in wavelength shift against the change in curvature of the sensor. As can be seen, the lower wavelength attenuation band had a bend sensitivity of $\mathrm{d} \lambda / \mathrm{dR}=22.3 \mathrm{~nm} . \mathrm{m} \pm 0.14$ and the higher attenuation band had a bend sensitivity of $\mathrm{d} \lambda / \mathrm{dR}=-19.1 \mathrm{~nm} . \mathrm{m} \pm 0.3$; shown by the trend lines. This sensitivity is the highest seen for this fibre to the best of the authors' knowledge. 


\subsection{SPECTRAL TEMPERATURE CHARACTERISTICS}

The temperature sensitivity was investigated by placing the LPG on an insulated Peltier cooler. Varying the Peltier temperature over a $80{ }^{\circ} \mathrm{C}$ range resulted in the attenuation bands converging as with the bending response. Fig. 5. shows the temperature sensitivity of the attenuation bands. The temperature sensitivity of the band centred on $1540 \mathrm{~nm}$ was $\mathrm{d} \lambda / \mathrm{dT}=0.54 \mathrm{~nm}^{\circ} \mathrm{C}^{-1}$, which was twice the sensitivity of the band centred on $1646 \mathrm{~nm}$, where $\mathrm{d} \lambda / \mathrm{dT}=-0.28 \mathrm{~nm}{ }^{\circ} \mathrm{C}^{-1}$.

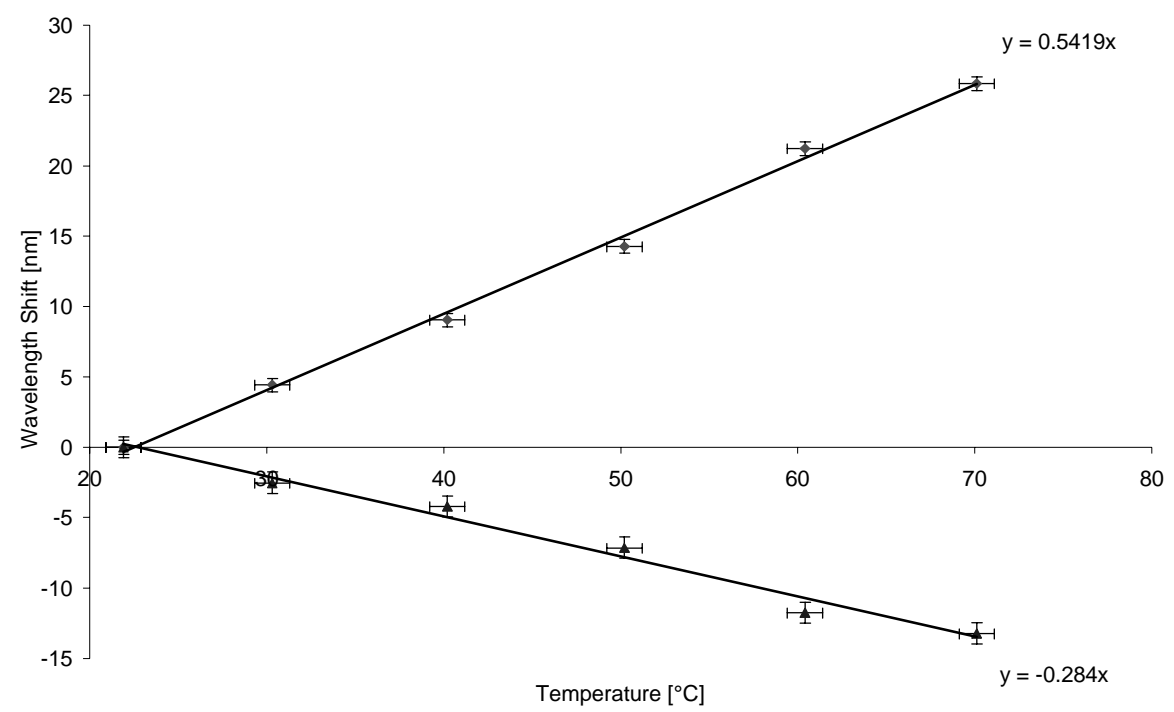

Fig. 5. Temperature Sensitivity of the attenuation bands of the LPG at 1540/1640nm.

The experimental results for the bending and temperature show a high spectral sensitivity of the LPG to the external measurands.

\section{THEORETICAL MODELLING}

The resonance wavelength of attenuation bands of an LPG with a period $\Lambda$ are determined by the phase-matching condition

$$
\delta n_{e f f} \Lambda=\lambda
$$

where

$$
\left[n_{\text {eff } f_{\text {core }}}{ }^{01}\left(\lambda_{l}\right)-n_{\text {eff }_{\text {cladding }}}{ }^{1 v}\left(\lambda_{i}\right)\right]_{i}=\delta n_{\text {eff }}
$$

Thus investigating the associated cladding mode for a given attenuation band provides the spectral response of the attenuation band. The number and the spectral properties of cladding modes are determined by the fibre structure [1012]. The geometrical dependence is not apparent from equations (2) and (3), but is taken into account in deriving the dispersion relation for a given refractive index profile [12]. The properties of individual cladding modes may be observed through their associated attenuation bands.

In the work reported here, the values of the core and cladding modes effective $\left(n_{c o} 01, n_{c l}{ }^{V}\right)$ and group $\left(n_{c o g}, n_{c l g}\right)$ indices were obtained by solving numerically the dispersion relationship for a 4-layered fibre using the technique described by Yeh \& Lindgren [12]. The predicted values were confirmed using Tsao's expressions for a dispersion relationship for a 4-layered fibre [11]. 
The above dispersion relationships define the $\mathrm{LP}_{01}$ core mode and the $\mathrm{HE}_{1 \mathrm{v}}$ axially symmetric cladding modes. We are assuming here that the grating consists of a circularly symmetric index perturbation transverse to the axis of the fibre, so that the only non-zero coupling coefficients between the core mode and the cladding modes involve cladding modes of azimuthal order 1 [10].

However, the length and small asymmetry of a LPG can allow coupling to the azimuthal order 2 cladding modes. The following theoretical results are based upon the azimuthal order 2.

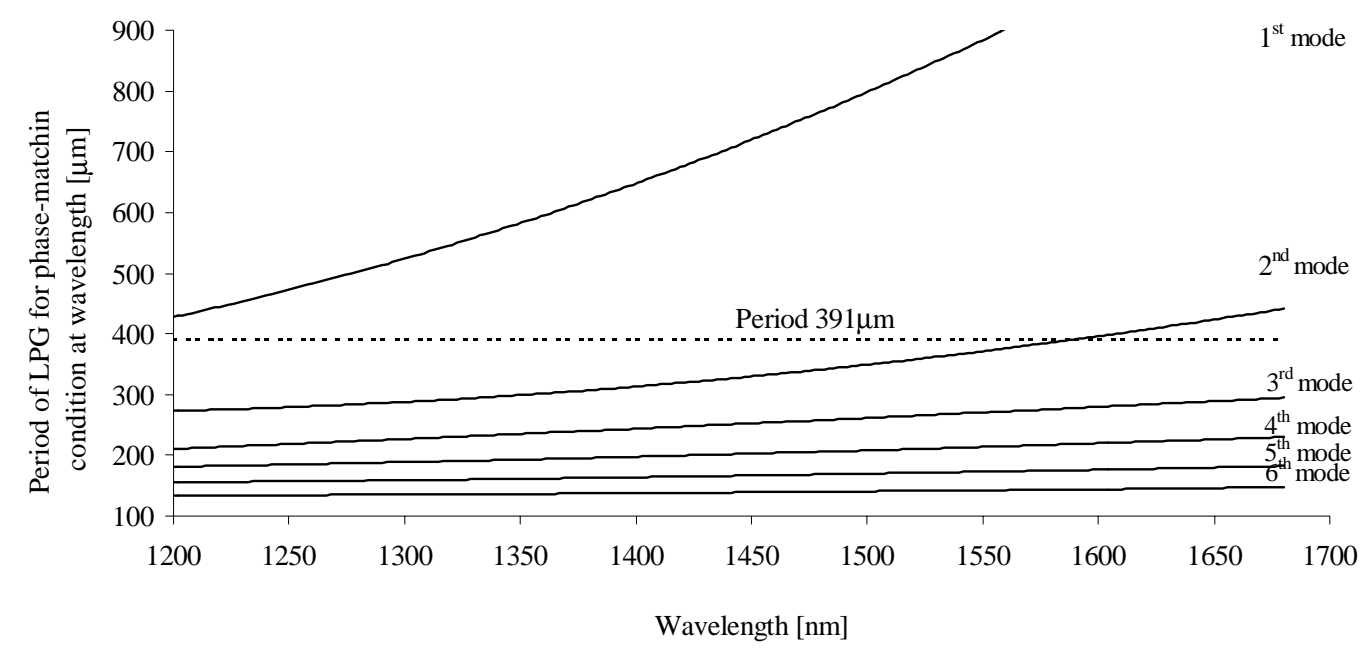

Fig. 6. Theoretical modelling of the phase matching curves for the first six $\mathrm{HE}_{\mathrm{EH}} \mathrm{EH}_{2, \mathrm{n}}$ cladding modes.

This shows theoretically that a long period grating with a period of $391 \mu \mathrm{m}$ has a phase-matching condition with the $2^{\text {nd }}$ cladding mode of the $2^{\text {nd }}$ azimuthal order at approximately $1600 \mathrm{~nm}$.

The experimental results showed that the transmission spectrum of a LPG fabricated with a period of $391 \mu \mathrm{m}$ had dual resonance attenuation bands centrally located at around $1595 \mathrm{~nm}$, giving reasonable agreement with the theoretical prediction.

\subsection{SPECTRAL CHARACTERISTICS OF THE DUAL RESONANCE ATTENUATION BANDS.}

There are both blue and red wavelength shifts observed for curvature and temperature variations experienced by the LPG. This behaviour can be modelled in two ways.

The first way involves looking at the group refractive indices of the core and cladding modes. We define the differential

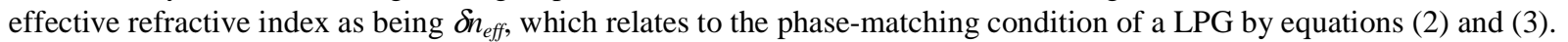
To simplify the analysis the ordinal $v$, which represents the order of the cladding mode, has been dropped. Since the $\delta n_{e f f}$ is a function of the operating wavelength, differentiating both sides of the above equation with respect to wavelength yields:

$$
\frac{d \Lambda}{d \lambda}=\frac{\delta n_{\text {eff }}-\lambda\left[\frac{d\left(\delta n_{\text {eff }}\right)}{d \lambda}\right]}{\left(\delta n_{\text {eff }}\right)^{2}}
$$

The group index of the core mode $\left(n_{c o g}\right)$ and the cladding modes $\left(n_{c l g}\right)$ are defined as: 


$$
n_{c o g}=n_{e f f_{c o r e}}-\lambda \frac{d n_{\text {effcore }}}{d \lambda}
$$

and

$$
n_{\text {clg }}=n_{\text {eff } f_{\text {cladding }}}-\lambda \frac{d n_{\text {eff }} \text { cladding }}{d \lambda}
$$

Substituting expressions (5) and (6) into (4), an expression for the slope of the coupling wavelength versus grating period curve is obtained [13]:

$$
\frac{d \Lambda}{d \lambda}=\frac{\delta n_{g}}{\left(\delta n_{e f f}\right)^{2}}
$$

where $\delta n_{g}=n_{c o g}-n_{c l g}$.

The above relationship (7) demonstrates that the slope of the coupling wavelength versus period is dependent upon both the differential effective index between the core mode and the cladding mode, $\delta n_{e f f}$, and also the differential group index, $\delta n_{g}$. The polarity of the slope of the above relationship at a given coupling wavelength is the same as the polarity of the differential group index, $\delta n_{g}$. The differential group index value can be either positive or negative. If the differential group index is positive at the phase-matching condition then the core group index is greater than the cladding group index. Alternatively if the differential group index is negative then the opposite is true: the cladding group index is greater than core group index.

The differential group index, $\delta n_{g}$, dictates the spectral behaviour of the attenuation band providing a blue/red wavelength shift. This can be seen using expression (2) written in the form $\Delta \beta=\frac{2 \pi}{\Lambda}$ to give:

$$
\frac{d(\Delta \beta)}{d \lambda}=2 \pi \frac{d}{d \lambda}\left(\frac{1}{\Lambda}\right)=\frac{-2 \pi}{\Lambda^{2}}\left(\frac{d \Lambda}{d \lambda}\right)
$$

Thus a blue spectral shift in the attenuation band $\frac{d(\Delta \beta)}{d \lambda}>0$ would be observed when cladding group index is greater than the core group index at the phase-matching condition, whilst a red spectral shift in the attenuation band $\frac{d(\Delta \beta)}{d \lambda}<0$ would be observed when core group index is greater than the cladding group index at the phase-matching condition.

The differential group index, $\delta n_{g}$, is dependent upon the properties of the host fibre as well as the period of the LPG (the phase-matching condition). 


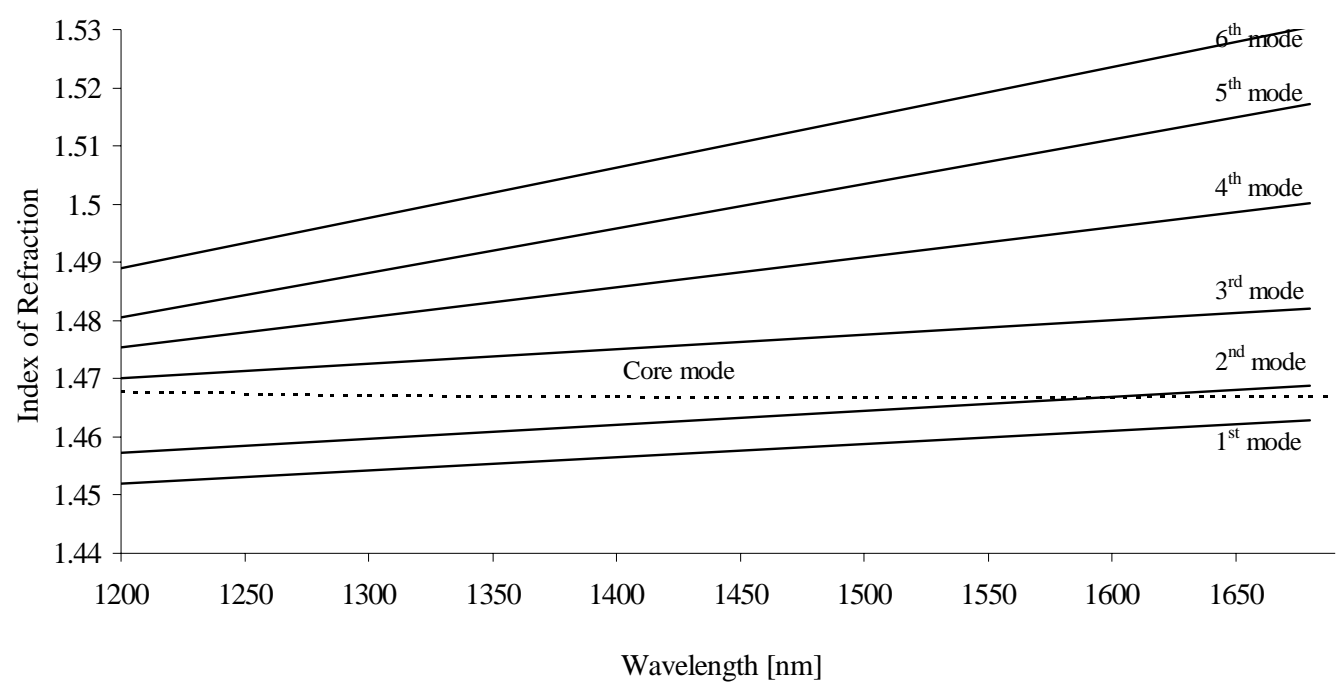

Fig .7. A comparison between the group index of refraction of various cladding modes and the core mode of the PTL optical fibre.

In Fig. 7 the variation of the group refractive index of various order cladding modes is compared to the group refractive index of the core, to indicate where we might expect a blue or red spectral shift.

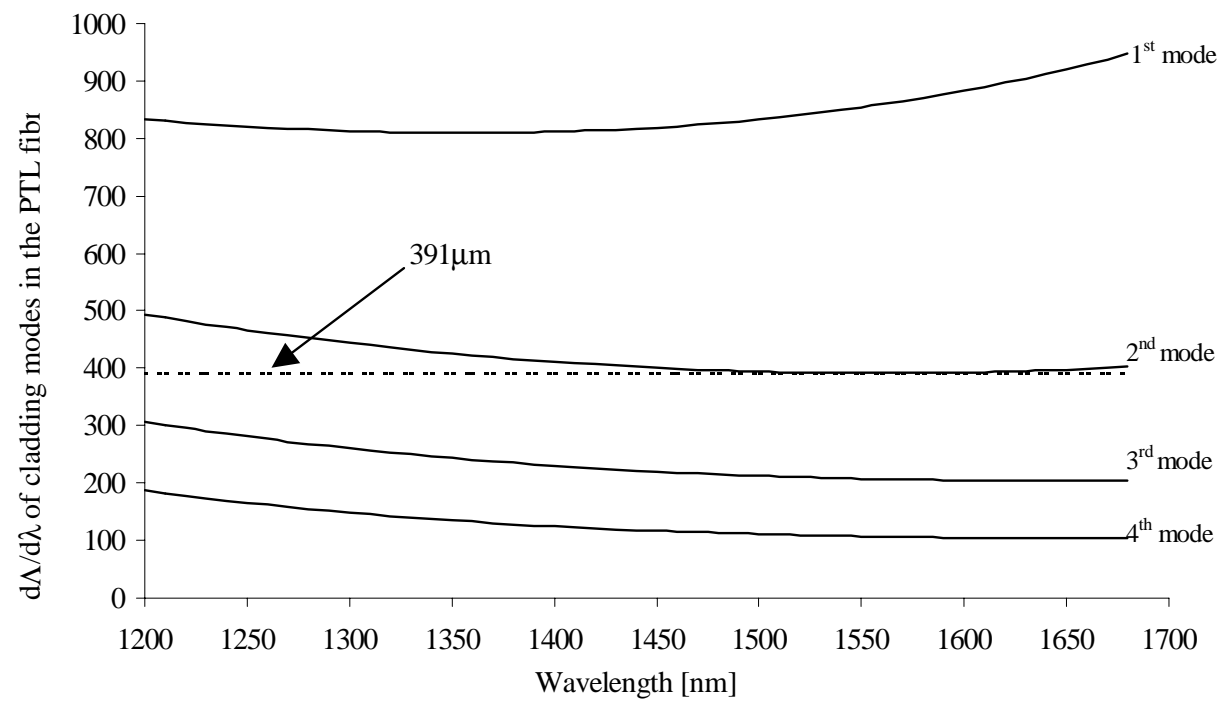

Fig. 8. Shows that the grating period $391 \mu \mathrm{m}$ has two attenuation bands associated with the $2^{\text {nd }}$ cladding mode.

Fig. 8. Shows that a LPG with a $391 \mu \mathrm{m}$ period has two phase matching conditions located either side of $1600 \mathrm{~nm}$, thus showing dual resonance attenuation bands associated with the $2^{\text {nd }}$ order, $\mathrm{HE} / \mathrm{EH}_{2, \mathrm{n}}$ mode. 


\subsection{SENSING PARAMETER}

The sensing parameter, which is defined as $\frac{d \lambda}{d \delta n_{\text {eff }}}$ [14], can be expressed as a function of the difference between the differential effective index and the differential group index:

$$
\frac{d \lambda}{d \delta n_{\text {eff }}}=\frac{\lambda}{\left(\delta n_{\text {eff }}-\delta n_{g}\right)}
$$

The sensing parameter relates to the sensitivity of the attenuation band. Fig. 9 shows that the parameter associated with the 2 nd cladding mode changes from a red wavelength shift at $1590 \mathrm{~nm}$ to a blue wavelength shift at $1630 \mathrm{~nm}$.

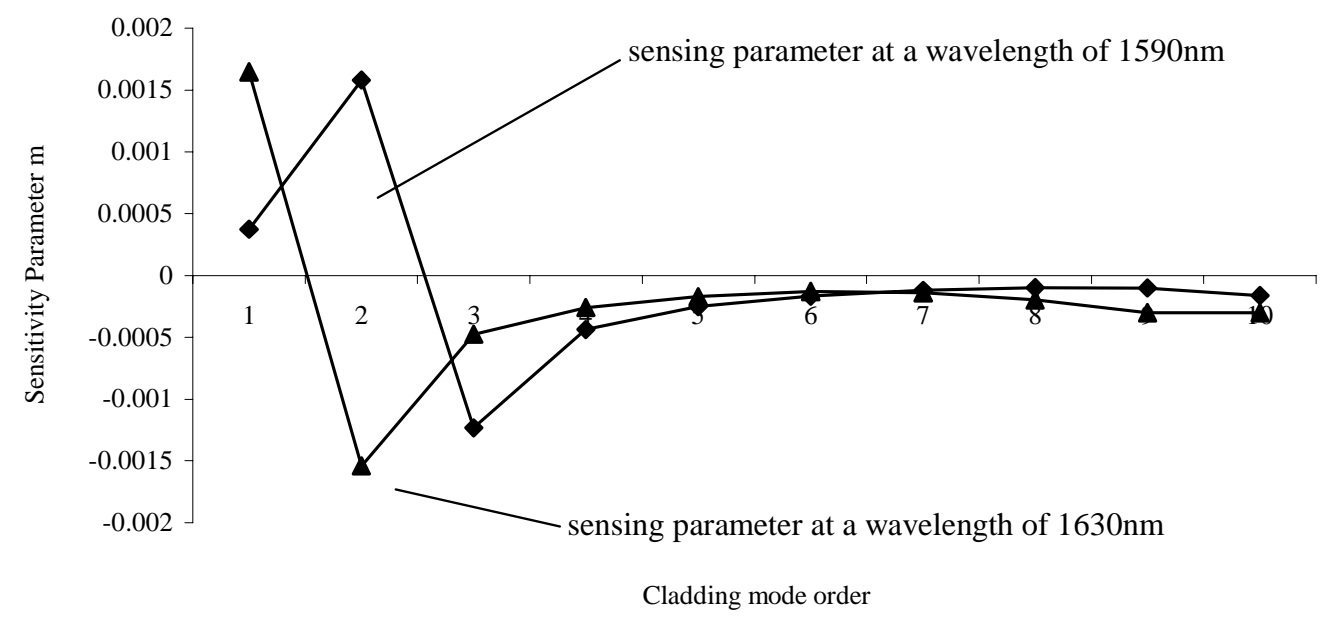

Fig. 9. Sensing parameter vs cladding mode order showing an enhanced sensitivity region associated with the TP of the $2^{\text {nd }}$ order mode.

Discriminating between the temperature and bending sensitivities of the two attenuation bands using the inverse matrix method, results in a condition number of 230 , which is a reasonable value compared to other discriminatory sensors [1517].

\section{CONCLUSIONS}

A long period grating with a period of $391 \mu \mathrm{m}$ was written into a progressive three layered optical fibre. The resulting attenuation bands were found to include a pair located either side of a particular modal turning point. These bands had increased sensitivity to bending with the higher wavelength band having a sensitivity of $-19.1 \mathrm{~nm}$.m and the lower wavelength band being $+22.3 \mathrm{~nm}$. $\mathrm{m}$. This is the highest seen for this fibre to the best of the authors' knowledge.

The attenuation bands were found to have significantly different temperature sensitivities with the band centred on $1540 \mathrm{~nm}$ having a sensitivity of $\mathrm{d} \lambda / \mathrm{dT}=0.54 \mathrm{~nm}{ }^{\circ} \mathrm{C}^{-1}$, which was twice that of the band centred on $1646 \mathrm{~nm}$, where $\mathrm{d} \lambda / \mathrm{dT}=-0.28 \mathrm{~nm}^{\circ} \mathrm{C}^{-1}$.

Theoretical analysis of the dual attenuation bands has shown the bands to be associated with the second order cladding modes $\mathrm{HE} / \mathrm{EH}_{2, \mathrm{n}}$ and provides reasonable agreement with the experimental data.

Due to the three layered structure of the fibre, the grating characteristics are independent of the external refractive index hence allowing the grating to be embedded into a composite without affecting the grating performance. 


\section{ACKNOWLEDGMENTS}

The authors would like to thank Fibrecore Limited, in particular T. Hart, for supplying the PTL fibre and its specification.

\section{REFERENCES}

1. A.M. Vengsarkar, J.R. Pedrazzani, J.B. Judkins, P.J. Lemaire, N.S. Bergano and C.R. Davidson. 'Long-period fibre-grating-based gain equalizers', Opt. Lett., 1996, 21, (5) pp. 336-338

2. A.M. Vengsarkar, P.J. Lemaire, J.B. Judkins, V. Bhatia, T. Erdogan and J.E. Sipe. 'Long-period fiber gratings as band rejection filters', J. Lightwave Technol., 1996, 14, pp. 58-64

3. H.J. Patrick, A.D. Kersey, F. Bucholtz, 'Analysis of the response of long period fibre gratings to external index of refractive', J. Lightwave Technol., 1998, 16, (9) pp 1606-1612

4. V. Bhatia, A.M. Vengsarkar, 'Optical fibre long-period grating sensors', Opt. Lett., 1996, 21, (9) pp. 692-694

5. H.J. Patrick, C.C. Chang and S.T. Vohra, 'Long period grating for structural bending sensing', Electron. Lett., 1998, 34, (18) pp. 1773-1775

6. T. Allsop. D.J. Webb and I Bennion. 'Bend and index insensitive long period grating in progressive three layered optical fibre', Electron. Lett., 2003, 39, pp 508-509

7. X. Shu, L. Zhang and I. Bennion. 'Sensitivity Characteristics of Long Period Fiber Gratings.' J. Lightwave Technol., 2002, 20, pp 255-266

8. B.H. Lee and J. Nishii, 'Self-interference of long-period fibre grating and its application as temperature sensor', Electron. Lett., 1998, 34, (21) pp. 2059-2060

9. W. Du, H. Tam, M. Liu, and X. Tao. 'Long-period fiber grating bending sensors in laminated composite structure', SPIE Conf. Proc. Smart structures and materials, SPIE, San Diego, 1998, Vol. 3330, pp 284-292

10. T. Erdogan, "Cladding-mode resonances in short- and long-period fiber Grating filters", J. Opt. Soc. Am. A., vol.4, no.8, pp.1760-1773, 1997.

11. C. Tsao, Optical Fibre Waveguide Analysis. New York: Oxford, 1992.

12. C. Yeh, G. Lindgren, "Computing the propagation characteristics of radially stratified fibers: an efficient method", Appl. Opt. vol. 16, no.2, pp.483-493, 1977

13. V. Bhatia, Properties and sensing applications of long period gratings, (PhD thesis, Virginia tech, Blacksburg, Virginia, 1996)

14. T. Allsop, D.J. Webb and I. Bennion, 'A comparison of the sensing characteristics of long period gratings written in three different types of fiber', Opt. Fiber Tech. 2003, 9, pp 210-223

15. T. Allsop et al, 'Bending and Orientation Characteristics of Long period Gratings Written in D-Shaped Optical Fiber', IEEE Trans. Instum. Meas., 2004, 53 (1) pp 130-135

16. H. Patrick, G. Williams, A. Kersey, J. Pedrazzani, and A. Vengsarkar, 'Hybrid fiber bragg grating/long period fiber grating sensor for strain/temperature discrimination,' Photon. Technol. Lett., 1996, 8 (9) pp 1223-1225

17. M. G. Xu, J.-L. Archambault, L. Reekie, and J. P. Dakin, "Discrimination between strain and temperature effects using dual-wavelength fiber grating sensors," Electron. Lett., 1994, 30, (13), pp. 1085-1087 